\title{
Direct differentiation of Dental Pulp Pluripotent-like Stem Cells differentiation into Hepatocyte-like Cells
}

Carlos Gil-Recio ${ }^{1}$, Sheyla Montori ${ }^{1}$, Cámara Vallejo ${ }^{2}$, Saddam Al Demour ${ }^{3}$, E. Ferrés-Padró ${ }^{4}$, Miguel Barajas ${ }^{5}$, Carles Martin ${ }^{2}$, Ashraf Al Madhoun ${ }^{6 *}$ and Maher Atari ${ }^{1,7^{*}}$

${ }^{1}$ Regenerative Medicine Research Institute, UIC Barcelona, Barcelona, Spain

${ }^{2}$ Oral and Maxillofacial Surgery Department, Hospital Clinico de Barcelona, Barcelona, Spain

${ }^{3}$ Department of Special Surgery/Division of Urology, The University of Jordan, School of Medicine, Amman, Jordan.

${ }^{4}$ Oral and Maxillofacial Surgery Department, Fundació Hospital de Nens de Barcelona, Barcelona, Spain

${ }^{5}$ Biochemistry and Molecular Biology Department, Universidad Pública de Navarra, Pamplona, Spain

${ }^{6}$ Department of Genetics and Bioinformatics, Functional Genomic Unit, Dasman Diabetes Institute, Kuwait

${ }^{7}$ Surgery and Oral Implantology Department, College of Dentistry Universitat Internacional de Catalunya, Spain

\section{Running Title:}

Hepatocyte generation from DPPSCS

\section{Address for Correspondence:}

*Prof. Maher Atari, Director of UIC Regenerative Medicine Research Institute, MedicalSurgical Pathology Department, Universitat Internacional de Catalunya, St Josep Trueta, sn08 195, Sant Cugat del Vallès, Barcelona, Spain. Telephone: +34 935042030

(ext 5824). E-mail; matari@biointelligentsl.com

And

*Ashraf Al Madhoun, Department of Genetics and Bioinformatics, Functional Genomic Unit, Research Division, Dasman Diabetes Institute, Dasman 15462, Kuwait.

Telephone: +965 22242999 (ext. 2805). E-mail: ashraf.madhoun@dasmaninstitute.org, 


\begin{abstract}
Liver diseases is a major cause of morbidity and mortality. Dental pulp pluripotent-like stem cells (DPPSC) are a considerable promise in tissue engineering and regenerative medicine as a source of tissue-specific cells, therefore the aim of this study is to demonstrate their ability to generate functional hepatocyte-like cells in vitro. Cells were cultured in serum free- differentiation media supplemented with growth factors and cytokines to direct the differentiation into hepatocytes in a process that recapitulate liver development. At day 5, the differentiated DPPSC cells expressed the endodermal markers FOXA1 and FOXA2. Then, the cells were derived into hepatic lineage generating hepatocyte-like cells. In addition to the associated morphological changes, the cells expressed the hepatic genes HNF6, AFP. The terminally differentiated hepatocyte-like cells expressed the liver functional proteins albumin and CYP3A4. In this study, we report an efficient serum free protocol to differentiate DPPSCs into functional hepatocyte like cells. Our approach promotes the use of DPPSCs, as new source of stem cells, for prospective use in liver regenerative medicine.
\end{abstract}

Keywords: hepatocyte differentiation, adult stem cells, dental pulp pluripotent stem cells, DPPSCs

Abbreviations

AST: Aspartate aminotransferase, BAL: Bioartificial liver, CGH: Chromosome genomic, hybridization, DEX: Dexamethasone, DPMSC: Dental pulp mesenchymal stem cell, DPPSC: Dental pulp pluripotent stem cells, EB: Embryoid bodies, ECM: Extracellular matrix, ESDL: End-stage liver disease , GGT: Gammaglutamyl transferase, HB: Hepatoblasts, HCC: Hepatocellular carcinoma, HT: Hepatocyte 
transplantation, MSC: Mesenchymal stromal cells, OLT: Orthotopic liver transplantation, OSM: Oncostatin M

\section{Introduction}

The liver is the largest internal organ providing essential metabolic, exocrine and endocrine functions. Hepatocytes are parenchymal cells which comprise approximately $70 \%$ of the adult liver mass. Both hepatocytes and cholangyocytes, the bile duct epithelial cells, are derived from the embryonic endoderm (Zorn 2008). The liver is the main homeostasis regulator; thus, liver diseases cause high morbidity and mortality rates. Many hepatic disorders lead to end-stage liver diseases (ESLD) and liver failure. The incidence of ESLD is increasing worldwide (Blachier Leleu et al. 2013), and the current optimal treatment for ESLD is orthotopic liver transplantation (OLT) which is nowadays a routine procedure with five-year survival for more than $70 \%$ of the patients (Dawwas Gimson et al. 2007). However, the main obstacle in liver transplantation is the growing discrepancy between the organ demand and donors availability. In fact, $15 \%$ listed patients die while awaiting a liver graft (Wertheim Petrowsky et al. 2011). Considering, the shortage of liver to be transplanted, hepatocyte transplantation (HT) is a promising alternative to OLT for many clinical applications (Wang Zhou et al. 2014). The procedure is less invasive than OLT and can be performed repeatedly. It can be especially useful in liver-based inborn errors of metabolism where the aim is to replace a single deficient enzyme or its product (Wang Zhou et al. 2014). At present, hepatocyte transplantation is also donor availability dependent. Therefore, the scientific community has put a lot of effort into obtaining hepatocyte-like cells from sources other than the human adult liver. 
Earlier, strategies are based on the use of human embryonic stem cells (hESC) as the only prospective source for the generation of hepatocyte-like cells; however, other sources of stem cells, including the induced pluripotent stem cells (iPSCs) and bone marrow mesenchymal stem cells (BM-MSCs) are possible alternatives. The differentiation of hESC into hepatocytes is first demonstrated by Rambhatla and his team (Rambhatla Chiu et al. 2003). Since then, many studies have focused on enhancing culture conditions to obtain homogeneous cell population. The knowledge acquired from hESC hepatogenesis are successfully applied to iPSCs (Si-Tayeb Noto et al. 2010; Chen Tseng et al. 2012). For clinical usage, hESC and iPSCs has common limitations, such as, ethical complications and genetic manipulation. Several protocols are revealed vulnerable results, inefficient differentiation and lack of maturation, low yield and heterogeneous cell populations. Furthermore, teratoma formation due to immortalized undifferentiated cells is considered as the main obstacle in the use of hESC and iPSCs (Fu \& Xu 2012).

Reports for the generation of hepatocyte-like cells from different sources of adult stem cells have been published including MSCs isolated from the bone marrow (Lee Kuo et al. 2004; Banas Teratani et al. 2007), adipose tissue (Seo Suh et al. 2005) or the umbilical cord blood (Hong Gang et al. 2005; Kang Zang et al. 2005). The Human bone marrow multipotent adult progenitor cells (MAPC) differentiate into hepatocyte-like cells expressing CK19, AFP, CK18, HepPar-1, and CD26 proteins; also these cells are reported to produce albumin, urea, and glycogen (Schwartz Reyes et al. 2002). Nevertheless, unlike embryonic stem cells, studies lack critical characterization of adult stem cells- mediated hepatocyte-like cells including non-consistent percentage of the generated hepatocyte-like cells, variations in the hepatic markers usage and no characterization for the remaining population during the differentiation process. 
In previous studies our group has described a new population of adult stem cells named dental pulp pluripotent-like stem cells (DPPSCs) (Atari Barajas et al. 2011; Atari GilRecio et al. 2012; Nunez-Toldra Dosta et al. 2017). These cells are isolated from the third molar - dental pulp and were found to express the pluripotency markers OCT3/4, NANOG and SOX2 (Atari Barajas et al. 2011). Similar to ESCs, DPPSCs generate cells from each of the three germ-embryonic layers (Atari Barajas et al. 2011; Atari CaballeSerrano et al. 2012), including bone-, endothelial- and neural- like cells. In this study, we aimed to differentiate DPPSCs into mature- functional hepatocyte-like cells. Toward this aim; serum free- directed- differentiation protocol was evaluated. DPPSCs were first differentiated into definitive endoderm (DE) then pre-hepatocyte-like cells were generated which were induced toward mature functional cells. At each differentiation step, the identity of the generated cells was determined identifying stage-specific makers and optical observation. Moreover, functional analyses were used to confirm the maturation of the differentiated hepatocyte-like cells including albumin secretion, glycogen storage and cytochrome P450 3A4 activity (CYP3A4). 


\section{Material and methods}

\section{DPPSCs Culture and Maintenance}

In this study, the used DPPSCs clones were previously isolated and characterized by our group as previously described (Atari Gil-Recio et al. 2012; Nunez-Toldra MartinezSarra et al. 2017). In Brief, immediately after extraction, the third molars were washed using gauze soaked in $70 \%$ ethanol and sterile distilled water. The molar pulp tissues were extracted from the dental pulp using a nerve-puller file 15 and forceps. Cells were isolated by digesting the pulp tissue with collagenase type I ( $3 \mathrm{mg} / \mathrm{ml}$, Sigma) for 60 minutes at $37^{\circ} \mathrm{C}$. Then, cells were then separated using an insulin syringe and centrifuged for 10 minutes at 1,800 rpm. The cell fraction was washed twice with $1 \mathrm{X}$ PBS. Once collected, the cells were counted and seeded in DPPSC medium. Primary cell lines were established, medium was changed every 4 days. During the splitting/passages of DPPSCs, cell density was maintained at $80-100$ cells $/ \mathrm{cm}^{2}$. DPPSCs were cultured in pre-coated flasks with $100 \mathrm{ng} / \mathrm{ml}$ Fibronectin (Life Technologies) in a medium consist of 60\% DMEM-low glucose (Life Technologies) and 40\% MCDB-201 (Sigma-Aldrich); supplemented with 1X SITE Liquid Media Supplement (SigmaAldrich); $1 \mathrm{X}$ linoleic acid-bovine serum albumin (LA-BSA, Sigma-Aldrich); $10^{-4} \mathrm{M} \mathrm{L-}$ ascorbic acid 2-phosphate (Sigma-Aldrich); 1X Penicillin-Streptomycin (PAA); 2\% fetal bovine serum (FBS) (Sigma-Aldrich); $10 \mathrm{ng} / \mathrm{ml}$ hPDGF-BB (Abcam); $10 \mathrm{ng} / \mathrm{ml}$ EGF (Sigma-Aldrich); $1000 \mathrm{U} / \mathrm{ml}$ LIF (Millipore); chemically defined Lipid Concentrate (Life Technologies); $0.8 \mathrm{mg} / \mathrm{ml} \mathrm{BSA} \mathrm{(Sigma-Aldrich)} \mathrm{and} 55 \mu \mathrm{M} \beta$ mercaptoethanol (Sigma-Aldrich). 


\section{Endoderm Induction using Growth Factors}

For the endoderm induction, $5 \times 10^{4}$ cells $/ \mathrm{cm}^{2}$ were seeded on Collagen type I-coated 6well plates (CellCoat $\left.{ }^{\circledR}\right)$ and cultured in RPMI medium (Mediatech) containing GlutaMAX (Life technologies), penicillin/streptomycin, $0.5 \%$ defined fetal bovine serum (FBS; HyClone) and supplemented with each one of the following combinations of growth factors: (i) $100 \mathrm{ng} / \mathrm{ml}$ Activin A (Act A, R\&D Systems), (ii) $100 \mathrm{ng} / \mathrm{ml} \mathrm{Act} \mathrm{A}$ + 50 ng/ml Wnt3A (R\&D Systems), (iii) $100 \mathrm{ng} / \mathrm{ml} \mathrm{Act} \mathrm{A} \mathrm{+} 50$ ng/ml BMP4 (R\&D Systems), (iv) $100 \mathrm{ng} / \mathrm{ml}$ Act A $+50 \mathrm{ng} / \mathrm{ml} \mathrm{Wnt3A}+50 \mathrm{ng} / \mathrm{ml} \mathrm{BMP} 4$, (v) $100 \mathrm{ng} / \mathrm{ml} \mathrm{Act}$ A +10 ng/ml FGF4 (R\&D Systems) or (vi) 100 ng/ml Act A +10 ng/ml $\beta F G F$ (Life technologies). Three days post-induction, the medium was replaced with the same RPMI-based medium but replacing FBS with 2\% knock-out serum replacement (KOSR, Life technologies) and incubation was continued for another 2 days.

\section{Endoderm Induction Using Different Biomaterial}

For the control group, $5 \times 10^{4}$ cells $/ \mathrm{cm}^{2}$ were seeded on Collagen type I-coated 6-well plates (CellCoat $\left.{ }^{\circledR}\right)$ placed in RPMI medium (Mediatech) supplemented with GlutaMAX (Life technologies) and penicillin/streptomycin and containing $0.5 \%$ defined FBS and $100 \mathrm{ng} / \mathrm{ml}$ Act A (R\&D Systems). Three days post-induction, the medium was refreshed using the same RPMI-based medium but replacing FBS by KOSR 2\% for two more days. Three additional groups were cultured under the same conditions as the control: (i) cells were seeded on plates pre-coated with $100 \mathrm{ng} / \mathrm{ml}$ Fibronectin (FN), (ii) $0.5 \%$ FBS was replaced by $2 \%$ KOSR during the first 3 days; (iii) Immediately after seeding, cell were co-cultured with 20,000 HepG2 cells $/ \mathrm{cm}^{2}$. 


\section{Hepatogenic differentiation}

Following the identification of the optimal conditions for definitive endoderm (DE) induction; we perform the procol desctibed in Fiure 1 to generate hepatocytes-like cells, we followed the protocol described in the schematic differentiation protocol supplemental figure (6). DE- induction was performed as described earlier using 100 $\mathrm{ng} / \mathrm{mL}$ Act $\mathrm{A}$ as the main supplemental cytokine. The Hepatocyte specification was initiated using RPMI medium supplemented with 2\% KOSR, 10 ng/ml FGF-4 and 10 ng/ml HGF (R\&D Systems). Three days later, RPMI was replaced with the enrich MEM medium (Sigma-Aldrich) with the same described supplements. 3- days later, hepatocyte maturation was induced using complete hepatocyte culture medium (HCM+ Singlequotes, Lonza) with all the supplements according to the manufacturer recommendations; containing $10 \mathrm{ng} / \mathrm{ml}$ FGF-4, 10 ng/ml HGF, 10 ng/ml Oncostatin M (OSM) and $10^{-7} \mathrm{M}$ dexamethasone (Dex; Sigma-Aldrich). The hepatocyte maturation was proceeded for 9 days, and the differentiation protocol was performed in a total of 22 days, the medium was refreshed every 2-3 days.

\section{Immunofluorescence}

Cells were seeded in on slides (Fisher Scientific). The cells were fixed with $4 \%$ paraformaldehyde (Sigma-Aldrich) for $15 \mathrm{~min}$ and permeabilized with $0.1 \mathrm{M}$ Triton X100 (Sigma-Aldrich) for $10 \mathrm{~min}$, at room temperature. Then, cells were blocked with 5\% BSA in PBS for 30 min and incubated for 1 hour in the primary antibodies: alpha-1 antitrypsin (AAT; Abcam), albumin (ALB; Abcam) or cytokeratin 19 (CK19; Abcam). Followed by 1 hour incubation with prospective secondary antibodies (Abcam). Between each step, the slides were washed with $1 \%$ BSA in PBS. Cells were examined 
using confocal fluorescence microscopy (Confocal 1024 microscope, Olympus AX70, Olympus Optical, Tokyo).

\section{Immunocytochemistry}

Cells were fixed with ThinPrep-CytoLyt Solution for $30 \mathrm{~min}$ at room temperature followed by centrifugation at $5,000 \mathrm{rpm}$ for $5 \mathrm{~min}$. The supernatant was discarded and the cell pellet was solubilized in Thinprep-CytoLyt Solution, centrifuged at $500 \mathrm{rcf}$ for 5 minutes and the pellet was solubilized now with PreservCyt (Thinprep) Solution for 15 min. Cellular collection was performed by a ThinPrep 2000 processor. The microscope slides were fixed for at least $30 \mathrm{~min}$ or for a maximum of $16 \mathrm{~h}$ in ethanol $96 \%$. After being washed twice with distilled water for $5 \mathrm{~min}$, the slides were then blocked in $0.5 \%$ hydrogen peroxide/methanol for $10 \mathrm{~min}$. The immunostaining was performed using a Leica MaxBond autostainer following the standard protocols given by the manufacturer. All products if not specified are from Novocastra. Washing steps were 3 for 1 min each with bond wash solution. The antigen retrieval procedure was performed according to Bond ${ }^{\mathrm{TM}}$ heat standard protocol (ER1) using citric buffer with $\mathrm{pH} 6$ for $30 \mathrm{~min}$ at $95^{\circ} \mathrm{C}$. The antibodies were (Alpha-fetoprotein) (AFP; Abcam) and AAT (Abcam). Cells were incubated for $15 \mathrm{~min}$ at room temperature. After that, 3 washing steps for 1 minute each with Bond wash solution. Then, slides were incubated with the post-primary for $8 \mathrm{~min}$ followed with 3 washing steps of 2 min each with bond wash solution. Next, slides were incubated in Polymer AP for 8 min followed by 2 washing steps of 2 min each with bond wash solution and 1 washing step of 1 min with distilled water. Then the Mixed Diaminobenzidine Refine was applied for $10 \mathrm{~min}$ and then for 5 more minutes. After 3 washing steps with distilled water for $1 \mathrm{~min}$ each, samples were counterstained with water haematoxylin for $5 \mathrm{~min}$ and washed with distilled water and wash bond solution 
respectively. Non-immune immunoglobulins of the same isotype as the primary antibodies were used as a control for each experiment.

PCR

Total RNA was isolated using Trizol Reagent (Life technologies) in accordance with the manufacturer's recommendations. cDNA was generated from 1-2 $\mu \mathrm{g}$ total RNA using the Transcriptor First Strand cDNA synthesis Kit (Roche). Real-time PCR was performed using the FastStart Universal SYBR Green Master (Roche). Values of gene expression were normalized to GAPDH. A list of the primers used for real time-PCR experiments are described in supplemental (S1) Table. For regular PCR TopTaq MasterMix kit (Qiagen) was used. Total liver RNA (Life technologies) or fetal liver RNA (Clontech) were used as controls. Cell lysate from iPSC were kindly supplied by Dr. Miguel Barajas.

\section{Hepatic Biochemical Analysis of supernatants}

Hepatic enzymatic profile for aspartate transaminase (AST) and gamma-glutamyl transferase (GGT) was analyzed from supernatants of the different samples. The activity was measured by specific colorimetric detection kits (Linear Chemicals) following the manufacturer's instructions. The specific compounds used for following the kinetics of reaction were as the following: for the GGT, the formation of 3-carboxy-4-nitroalinine was measured at 410nm; for the AST, the oxidation of NADH was measured at 340.

\section{Cytochrome P450 3A4 Metabolic Activity Assay}

Cytochrome P450 (CYP) 3A4 enzyme activity assay was assessed by measurement of luciferase activity with the P450-Glo CYP3A4 assay (Promega), per the manufacturer's instructions. Differentiated cells were treated with Rifampicin $(25 \mu \mathrm{M})$ for $48 \mathrm{hr}$. Then, 
cells were incubated at $37^{\circ} \mathrm{C}$ in fresh serum free medium containing $50 \mu \mathrm{mol} / \mathrm{L}$ Luciferin PFBE with or without $5 \mu \mathrm{M}$ Erythromycin (an inhibitor of the enzyme) for 30 min. Undifferentiated DPPSC were used as negative control. Later, $50 \mu \mathrm{L}$ of the medium was transferred into a 96-well plate, mixed with $50 \mu \mathrm{L}$ luciferin detection reagent to initiate the luminescent reaction. After 20 min incubation period at room temperature, the luminescence was measured with a luminometer (Biotek).

\section{Albumin Assay}

Conditioned medium was collected over from equivalent numbers of cells. The albumin secretion was measured by using an Albumin Fluorescence Assay Kit (Fluka) following the manufacturer's instructions. Briefly, first a calibration curve was done with different human albumin concentrations. These calibration samples were mixed with albumin blue 580 in 2-propanol diluted in buffer solution, then, the fluorescence was measured in a spectrofluoro-meter $(\lambda e x=600 \mathrm{~nm}, \lambda \mathrm{em}=630 \mathrm{~nm})$. Then, differentiated cells were mixed with the same reagents and the fluorescence was measured under the same conditions. Results are extrapolated to the calibration curve.

\section{Periodic acid-Schiff staining for glycogen accumulation}

Cells were fixed in $4 \%$ formaldehyde for $15 \mathrm{~min}$ at room temperature. After 2 washing steps with PBS, cells were incubated in $1 \%$ periodic acid for $5 \mathrm{~min}$, then washed with distilled water, and incubated with Schiff's reagent (Sigma-Aldrich) for $15 \mathrm{~min}$. After a 10 min wash in tap water, Karazi's haematoxylin counterstain was applied for $90 \mathrm{sec}$ and washed with water. 


\section{Statistical analysis}

Results are reported as an Mean \pm Standard Error of the mean (see figure legends for specific details regarding the number of biological replicates, independent experiments and technical replicates). Statistics were performed using the Statgraphics XVI software. The methods used were two-tailed Student's t-tests and ANOVA for multiple factors. Values with $\mathrm{p}<0.05$ were considered statistically significant.

\section{Ethics Statement}

All research in the present study was conducted in accordance with the code of ethics of the World Medical Association for experiments involving humans (Helsinki Declaration of 1975) and the guidelines on human stem cell research issued by the Committee of Bio-ethics at the Universitat Internacional de Catalunya. 


\section{Results}

\section{Definitive Endoderm Induction}

Using hESCs and iPSCs, studies has shown that Act A is the main DE induction factor. Therefore, we tested this concept on DPPSCs using Act A with or without other factors to mediate DE- differentiation. In addition to Act A signaling, we studied the influence of other endodermal induction pathways including Wnt3A, BMP4, $\beta \mathrm{FGF}, \mathrm{FGF} 4$ in the presence of Act A. the generation of DE- and early hepatocyte-like cells were monitored by the expression of FOXA1, FOXA2, GATA4 and AFP RNA and protein expressions. Relative to undifferentiated DPPSCs (Fig $1 \mathrm{G}$ and H), cells incubated with the cytokines showed morphological changes as detectable by optical microscopy. 5 days post DEinduction, cells cultured with media containing Act A (Fig 1A) alone, +Wnt3A (Fig 1B), +BMP4 (Fig 1C) or +Wnt3A and BMP4 (Fig 1D) showed similar cellular morphology. Nevertheless, incubation with Act + Wnt3A alone resulted in a lower cell growth. Cells cultured with Act + BMP4 (Fig 1C) had a uniform morphology but smaller size as compared to other treatments. In addition, Act A treatments with either $\beta$ FGF (Fig 1E) or FGF4 (Fig 1F) resulted in cellular elongation similar to that observed for fibroblast.

At molecular level, the expression of GATA4 transcripts was dramatically induced after 5 days treatment with Act $\mathrm{A}$ in the presence or absence of Wnt3A or BMP4 (Fig 1I). Cells cultured in media supplemented with Act with either $\beta$ FGF, FGF4 or Wnt3A + BMP4 did not show GATA4 upregulation as compared to undifferentiated DPPSCs.

The treatment with Act A and Wnt3A enhanced FOXA1 RNA-expression relative to controls and other treatments. Interestingly, treatments with Act A with or without BMP4 or FGF4 showed equivalent expression of FOXA1 with 2-3 fold increase relative 
to undifferentiated DPPSCs. Cells cultured media containing Act A with $\beta F G F$ or Wnt3A + BMP4 revealed non-significant upregulation of FOXA1 transcripts (Fig 1J). Surprisingly, the expression levels of FOXA2 transcripts were almost equivalently induced in all the described treatments with an average 2-5 folds higher that undifferentiated cells. (Fig 1K).

Interestingly, relative to undifferentiated DPPSCs, cellular treatment with Act A alone enhanced the expression of AFP RNA. With no further improvements in AFP transcript induction in cells treated with the other factors Wnt3A, BMP4 or FGF4 or their combinations (Fig 1L).

Accordingly, this study supports the importance of Act A alone or in combination with either Wnt3A or BMP4 in the induction of DE genes. However, Act A alone supplements was superior to induce the early hepatic marker AFP suggesting its role in hepatic cell specification. Thus, the following experiments were carried out using Act A alone for matters of simplicity.

In order to improve the differentiation protocol and study the influence of bio-materials on DPPSCs mediated DE- indication. Therefore, we carried out the differentiation under 4 different conditions: cells were cultured on plates pre coated with collagen I (Fig 2A), or fibronectin (Fig 2B), or collagen I in FBS-free media for 5 days (Fig 2C), or coculture with HepG2 cells (Fig 2D). DPPSCs cultured on Collagen I or Fibronectin showed a moderate growth rate and relativity similar morphological structure. As anticipated, day 5 culture on collagen I- defined FBS free media resulted in high cell death. On the other hand, DPPSCs co-cultured with HepG2 cells were over-grown and acquired a flat fibroblastic morphology.

Gene expression qRT-PCR study of the DE-genes FOXA1, FOXA2 and GATA4 revealed differentiation gene expression in response to the 4-cultural conditions. The 
expression of FOXA1 transcripts were 4-6 folds higher than undifferentiated cells; except for DPPSCs cells co-cultured with HepG2 cells which showed 2 fold increase in FOXA1 expression (Fig 2E). The gene expression of FOXA2 was higher in cells cultured on collagen I or fibronectin relative to other treatments (Fig 2F). On the other hand, GATA4 expression was equivalent in all treatments with minimal values detected in cells treated with FBS (Fig 2G).

In summary, DPPSCs cultured on the standard protocol using pre-coated collagen I or pre-coating with fibronectin were the cells to have the highest expression in all DEmarkers studied. Since, collagen I is the commonly used biomaterial to culture other stem cells, we restricted our study on the use of collagen I to induce cellular differentiation.

\section{Hepatic specification}

Using the standard DE- differentiation protocol, we proceeded to induce hepatic specification and induction using induction by incubating the generated DE- cells in a culture media containing FGF4 and HGF, Material and Methods section. The expression of several hepatic related markers after one and two weeks was detected (Fig 3A). 14-Days incubation in the standardized media, the expression of the hepatic markers was observed. In order to improve the expression of these genes at early stages of differentiation, cells were further incubated in a media supplemented with Act A for 7 days, instead of 5 days as described earlier. Interestingly, unlike the observation using standardized protocol (Fig 3A), the expression of the DE- and early hepatic markers, FOXA1 and AFP, respectively, was observed at day 7 in response to Act A prolong incubation period (Fig 3B). Accordingly, we proceed our experiments with a pro-long exposure to Act A, and studied the expression of several hepatogenic markers 
throughout the differentiation process. Real time gene expression studies showed that a peak expression of the endodermal gene FOXA1 at day 7 (Fig 3C). The expression of genes associated with hepatocyte maturation was observed at day 15 and beaked at day 22 post differentiation. The transcript expression of AAT, G6P and ALB showed 3-, 6and 1.5- folds increase on day 15 relative to undifferentiated DPPSCs (Fig 3D-F). The expression of these hepatic makers was further duplicated at day 22 of the differentiation, suggesting the maturation of the generated hepatocyte-like cells.

The genes expression modification during the differentiation process was associated with cellular structural changes toward hepatocyte-like morphology. At earlier stages, the cells were small and spindle-shaped. 7 days later, cell growth and elongation was observed (Fig 3G, J). After 2-weeks, the cells acquired epithelioid morphology (Fig 3H, K). After 22 days, polygonal cells were detected (Fig 3I, L).

Gene expression analysis revealed a distinct hepatocyte-like signature at day 22 of DPPSCs differentiation. In order to corroborate the qRT-PCR analysis and evaluate the differentiation efficiency, we ascertained the protein expression of the hepatic DE markers by immunofluorescence, immunocytochemistry and flow cytometric analysis.

Immunofluorescence analysis using specific antibodies directed against CK18, ALB and AAT proteins revealed a significant expression of the mature hepatic markers at day 22 of DPPSCs differentiation (Fig 4A-C). In addition, immunocytochemical analysis using AFP and AAT antibodies in the presence of Di-aminobenzidine and haematoxylin reagents demonstrated a significant expression of AFP and AAT proteins (Fig 4D-G).

Immunoreactivity studies revealed high expression of the early hepatic marker AFP, which inspired us to investigate the differentiation efficiency using flow cytometry, the 
differentiation protocol resulted in an enrichment of pre-hepatocyte-like cells, where $44 \%$ of the total cell count were positive to AFP protein at differentiation day 11, relative to cells treated with comparable $\mathrm{IgG}($ Fig $4 \mathrm{H})$.

\section{DPPSCs-derived hepatocyte-like cells demonstrate hepatic function:}

The enrichment for hepatocyte like cells encouraged us to test for prospective generation of functional mature hepatocytes. Our studies included glycogen storage, albumin secretion and the expression of active cytochromes

Mature hepatocyte are characterized with their ability to store glycogen. Interestingly, relative to undifferentiated DPPSCs (Fig 5A), most cells on Day 22 of differentiation were positive for periodic acid Schiff (PAS) staining indicative of glycogen storage, and the generation of mature hepatocytes (Fig 5B).

The metabolic cytochrome P450 3A4 (CYP3A4) is a hepatic enzyme mediates drug metabolism and reduces cytotoxicity. Notably, relative to undifferentiated cell, DPPSCs showed 5-fold increase in CYP3A4 enzymatic activity at day 22 of differentiation, which was significantly reduce in the presence of erythromycin (CYP3A4 inhibitor) (Fig 5C). Further, time course studies of the differentiated cells for gamma-glutamyl transferase (GGT) and aspartate transaminase (AST) activities revealed a time dependent upregulation of the liver metabolic enzymes which beaked at day 18 of differentiation (Fig 5D and E, respectively).

Albumin secretion was assayed with human albumin-specific fluorometric kit. As shown in figure $5 \mathrm{~F}$, at earlier stages of hepatogenesis, day 10 of differentiation, a limited amount of secreted albumin was detected in the media. However, upon maturation (day 18 and after), the amount of secreted albumin was doubled into 3.5 pg/cell. Taking these observations together; the described differentiation protocol (Fig6) 
efficiently induced generation of DPPSCs mediated functional hepatocyte-like cells and indicates that DPPSCs are useful cell model for hepatogenesis.

\section{Discussion}

The current strategy in stem cell differentiation protocols is to mimic cellular signaling events associated with the embryonic developmental process for the lineage of interest [8, 47, 48]. However, in-vitro cell differentiation gives a broad range of outcome lineages, thus, the development of a protocol that significantly improves a targeted cell type is of particular interest[49]. Targeted differentiation requires culturing the cells in chemically well-defined media, which regulate cell-signaling pathways, resulting in the generation of cell-type of interest. This approach have been successfully applied to hESCs and iPSCs [51-53], but have yet to be applied to other pluripotent cell types, including DPPSCs.

In this study, we applied an optimized 5-stages protocol directed to a stepwise differentiation of DPPSCs toward hepatocyte like cells. For efficient differentiation, cells were checked for each developmental stage using specific markers. Initially, DPPSCs were cultured for 5 days in a differentiation media supplemented with Act A, with or without Wnt3a, BMP4 or the growth factors $\beta$ FGF and FGF4. Interestingly, Act A alone was superior to induce DE- differentiation. Real time RT-PCR analysis revealed an increase in FOXA1, FOXA2 and GATA4 transcripts, the definitive endodermal markers. Accordingly, we restricted our protocol in the use of Act A alone. However, we observed that an additional 2-days culture in Act A containing media, dramatically activates the expression of FOXA1 and AFP, an early hepatogenesis marker. Our results are in accordance with the reported role of Act A inducing DE, in 
vitro, from pluripotent stem cells (D'Amour Agulnick et al. 2005; Wang McKnight et al. 2012). It is well known that Nodal signaling is required for endoderm specification during the gastrulation and Act $\mathrm{A}$ is the only source for such activity in vitro (Robert Lanza 2009). Further, DE- induction is dependent on duration of exposure to the stimulation factors; Agarwal et al., reported that 5-days incubation with Act A are sufficient to induce DE (Agarwal Holton et al. 2008). A recent study provided evidences that 7 days is an optimal incubation period to differentiate the cells into DElineage, which is associated with higher ALB expression during the hepatogenic specification (Toivonen Lundin et al. 2013). In this regard, we confirmed that 7 days is an ideal incubation period with Act A to mediate DPPSCs differentiation into DE.

During liver development, BMPs signaling is crucial; however, their functional role during in vitro- adult stem cell differentiation is not clear. Cultural media supplemented with BMP2 and BMP4 fail to improve hepatogenesis from adipose tissue stem cell (Bonora-Centelles Jover et al. 2009). Using hESCs, Act A mediated- DE induction was not adversely affected by the addition of BMP4 (Laflamme Chen et al. 2007). In accordance with the other studies, our results confirmed BMP4 signaling has no functional role during DPPSCs differentiation into DE reconciled by Act A induction. Alternatively, studies has shown that a combination of Act A and BMP4 signaling induce mesoderm formation (Laflamme Chen et al. 2007).

Media supplemented with Act A and Wnt3A have been widely used to induce DE (D'Amour Agulnick et al. 2005; Toivonen Lundin et al. 2013). Using hESCs, Mathew and his colleagues observed an enhanced DE induction using Act $\mathrm{A}+\mathrm{Wnt} 3 \mathrm{~A}$ supplemented media as compared to media contained Act A alone or in combination with BMP4 (Mathew Jaramillo et al. 2012). Similar to hESC, in this study, we observed 
an improved induction in DE- markers in DPPSCs cultured with Act A and Wnt3A relative to that cultured in media containing Act and growth factors $\beta$ FGF or FGF4.

The use of minimal amount of FBS in differentiation media has been previously reported to as an inhibitor for PI3K signaling, which is necessary to facilitate the activation of the supplemented Act A. Using 0.5\% FBS during the first 5 days of DE induction, D'Amour and her team observed an enhancement in the expression of Sox17 or FOXA2 in hESC (D'Amour Agulnick et al. 2005). In our protocol, we used 0.5\% FBS-media for the first 3 days of differentiation, then it was replaced with $2 \%$ KOSR, a serum that has been proven to be effective and suitable with defined components (Agarwal Holton et al. 2008). Nevertheless, the use of 2\% KOSR within the first 3 days was not sufficient to enhance DPPSCs differentiation into DE (DATA not shown).

As descripted in Figure 6, the growth factors FGF4 and HGF were used to induce hepatogenesis, followed by the usage of OSM with Dex for the hepatocytes maturation. The role of FGF4 and HGF in hepatocyte specification has been previously reported for both Wharton's jelly- and bone marrow mesenchymal stem cells (Schmidt Bladt et al. 1995; Schwartz Reyes et al. 2002; Kang Zang et al. 2005). In accordance, both FGF4 and HGF were required to mediate hepatic specification in DPPSCs (Figure 6). We observed an improved expression of several hepatic markers including immature and mature markers at day 13 of differentiation. The use of other FGF family members such as FGF2 and FGF1 has been reported to improve DE- induction and hepatic differentiation in hESC cells (Mathew Jaramillo et al. 2012). In the presence of Act A, $\beta$ FGF failed to improve DE- induction in DPPSCs, similar observations was reported for hESCs (Schwartz Linehan et al. 2005)

Studies have shown that the interleukine-6 family cytokine (OSM) is required for hepatocyte maturation in combination with glucocorticoids, such as Dex (Kamiya 
Kinoshita et al. 2001; Hanada Kayano et al. 2003). Addtionally, it is well documented that HGF, EGF and OSM have decisive effects on the maintenance of primary human hepatocytes in vitro (Dong Mandenius et al. 2008). The combination of HGF, OSM and Dex is widely used in protocols to differentiate stem cells into hepatocytes (Agarwal Holton et al. 2008; Li Yuan et al. 2014). In our protocol, we applied the maturation factors OSM and Dex at day 13, post the commitment to the hepatic fate. Consequently, we documented hepatocytes maturations using cellular and molecular techniques. After 15 days exposure to media containing OSM and Dex, we observed changes in cells morphology toward hepatocyte like structures, which was associated with ALB secretion, GGT and AST enzymatic activity.

After the establishment of the most optimal protocol used for DPPSC, we performed all the tests that were available for us in order to prove the efficacy of the differentiation of DPPSC into hepatocyte-like cells. At molecular level, differentiated DPPSC expressed AAT, ALB and G6P transcripts, genes that are markers for mature hepatocytes. Further, the protein expression of these markers was also documented in our study. Cellular morphological changes was also detected and mature cells acquired polygonal-shape typical for hepatocytes (Godoy Hewitt et al. 2013). In addition, DPPSCs mediated mature hepatocytes were functional. These cells stored glycogen, secreted ALB and other metabolic enzymes as efficient as other stem cells included MSCs [39] and hESCs(Ji Zhang et al. 2012; Subramanian Owens et al. 2014). Furthermore, differentiated DPPSCs have shown an improved Cyp3A4 enzymatic activity, suggesting that the generated hepatocytes can be used as a model for drug metabolism, a field where hepatocyte-like cells would be very useful for the pharmaceutical industry (Jozefczuk Prigione et al. 2011). 


\section{Conclusions}

We designed a novel protocol to differentiate DPPSCs into functional hepatocyte-like cells. Our protocol is a directed differentiation protocol mimicking the stepwise process observed during the embryonic development. Using DPPSCs as a stem cell model suggesting their potential usage for liver regenerative medicine and prospective treatments to be developed in the future. However, for clinical applications there are still many studies are needed to be improved, yet, the generated functional DPPSCshepatocyte-like cells can be used as a model for drug screening, hepatic metabolism studies and hepatic disease applications.

This contribution is funded by funded by the Universitat International de Catalunya (UIC) and the Agència de Gestió d'ajuts Universitaris i de Recerca, Generalitat de Catalunya (SGR 1060). The authors Carlos Gil-Recio, Ester Martínez-Sarrà Raquel Núñez-Toldrà were funded by the predoctoral grant Junior Faculty awarded by the Obra Social La Caixa and the UIC.

\section{ACKNOWLEDGMENTS}

This study was funded by the Universitat International de Catalunya (UIC) and the Agència de Gestió d'ajuts Universitaris i de Recerca, Generalitat de Catalunya project number SGR 1060 for MA, and Kuwait Foundation for the Advancement of Sciences (KFAS) under projects number RA-2013-009 for AAM. CGR, EMS and RNT were funded by the predoctoral grant Junior Faculty award from The Obra Social La Caixa and UIC.

\section{DISCLOSURES}

The authors declare no conflicts of interest. 
bioRxiv preprint doi: https://doi.org/10.1101/2020.12.09.418780; this version posted December 10,2020 . The copyright holder for this preprint (which was not certified by peer review) is the author/funder. All rights reserved. No reuse allowed without permission. 


\section{References}

Agarwal S, Holton KL, Lanza R (2008) Efficient differentiation of functional hepatocytes from human embryonic stem cells. Stem Cells 26(5), 1117-1127.

Atari M, Barajas M, Hernandez-Alfaro F et al. (2011) Isolation of pluripotent stem cells from human third molar dental pulp. Histol Histopathol 26(8), 1057-1070.

Atari M, Caballe-Serrano J, Gil-Recio C et al. (2012) The enhancement of osteogenesis through the use of dental pulp pluripotent stem cells in 3D. Bone 50(4), 930-941.

Atari M, Gil-Recio C, Fabregat M et al. (2012) Dental pulp of the third molar: a new source of pluripotent-like stem cells. J Cell Sci 125(Pt 14), 3343-3356.

Banas A, Teratani T, Yamamoto Y et al. (2007) Adipose tissue-derived mesenchymal stem cells as a source of human hepatocytes. Hepatology 46(1), 219-228.

Blachier M, Leleu H, Peck-Radosavljevic M, Valla DC, Roudot-Thoraval F (2013) The burden of liver disease in Europe: a review of available epidemiological data. $J$ Hepatol 58(3), 593-608.

Bonora-Centelles A, Jover R, Mirabet V et al. (2009) Sequential hepatogenic transdifferentiation of adipose tissue-derived stem cells: relevance of different extracellular signaling molecules, transcription factors involved, and expression of new key marker genes. Cell Transplant 18(12), 1319-1340.

Chen YF, Tseng CY, Wang HW, Kuo HC, Yang VW, Lee OK (2012) Rapid generation of mature hepatocyte-like cells from human induced pluripotent stem cells by an efficient three-step protocol. Hepatology 55(4), 1193-1203.

D'Amour KA, Agulnick AD, Eliazer S, Kelly OG, Kroon E, Baetge EE (2005) Efficient differentiation of human embryonic stem cells to definitive endoderm. Nat Biotechnol 23(12), 1534-1541.

Dawwas MF, Gimson AE, Lewsey JD, Copley LP, van der Meulen JH (2007) Survival after liver transplantation in the United Kingdom and Ireland compared with the United States. Gut 56(11), 1606-1613.

Dong J, Mandenius CF, Lubberstedt M et al. (2008) Evaluation and optimization of hepatocyte culture media factors by design of experiments (DoE) methodology. Cytotechnology 57(3), 251-261.

Fu X, Xu Y (2012) Challenges to the clinical application of pluripotent stem cells: towards genomic and functional stability. Genome Med 4(6), 55.

Godoy P, Hewitt NJ, Albrecht U et al. (2013) Recent advances in 2D and 3D in vitro systems using primary hepatocytes, alternative hepatocyte sources and nonparenchymal liver cells and their use in investigating mechanisms of hepatotoxicity, cell signaling and ADME. Arch Toxicol 87(8), 1315-1530.

Hanada S, Kayano H, Jiang J et al. (2003) Enhanced in vitro maturation of subcultivated fetal human hepatocytes in three dimensional culture using polyL-lactic acid scaffolds in the presence of oncostatin M. Int J Artif Organs 26(10), 943-951.

Hong SH, Gang EJ, Jeong JA et al. (2005) In vitro differentiation of human umbilical cord blood-derived mesenchymal stem cells into hepatocyte-like cells. Biochem Biophys Res Commun 330(4), 1153-1161.

Ji R, Zhang N, You N et al. (2012) The differentiation of MSCs into functional hepatocyte-like cells in a liver biomatrix scaffold and their transplantation into liver-fibrotic mice. Biomaterials 33(35), 8995-9008.

Jozefczuk J, Prigione A, Chavez L, Adjaye J (2011) Comparative analysis of human embryonic stem cell and induced pluripotent stem cell-derived hepatocyte-like 
cells reveals current drawbacks and possible strategies for improved differentiation. Stem Cells Dev 20(7), 1259-1275.

Kamiya A, Kinoshita T, Miyajima A (2001) Oncostatin M and hepatocyte growth factor induce hepatic maturation via distinct signaling pathways. FEBS Lett 492(1-2), 90-94.

Kang XQ, Zang WJ, Bao LJ et al. (2005) Fibroblast growth factor-4 and hepatocyte growth factor induce differentiation of human umbilical cord blood-derived mesenchymal stem cells into hepatocytes. World J Gastroenterol 11(47), 74617465.

Laflamme MA, Chen KY, Naumova AV et al. (2007) Cardiomyocytes derived from human embryonic stem cells in pro-survival factors enhance function of infarcted rat hearts. Nat Biotechnol 25(9), 1015-1024.

Lee KD, Kuo TK, Whang-Peng J et al. (2004) In vitro hepatic differentiation of human mesenchymal stem cells. Hepatology 40(6), 1275-1284.

Li X, Yuan J, Li W et al. (2014) Direct differentiation of homogeneous human adipose stem cells into functional hepatocytes by mimicking liver embryogenesis. J Cell Physiol 229(6), 801-812.

Mathew S, Jaramillo M, Zhang X, Zhang LA, Soto-Gutierrez A, Banerjee I (2012) Analysis of alternative signaling pathways of endoderm induction of human embryonic stem cells identifies context specific differences. BMC Syst Biol 6, 154.

Nunez-Toldra R, Dosta P, Montori S, Ramos V, Atari M, Borros S (2017) Improvement of osteogenesis in dental pulp pluripotent-like stem cells by oligopeptidemodified poly(beta-amino ester)s. Acta Biomater 53, 152-164.

Nunez-Toldra R, Martinez-Sarra E, Gil-Recio C et al. (2017) Dental pulp pluripotentlike stem cells (DPPSC), a new stem cell population with chromosomal stability and osteogenic capacity for biomaterials evaluation. BMC Cell Biol 18(1), 21.

Rambhatla L, Chiu CP, Kundu P, Peng Y, Carpenter MK (2003) Generation of hepatocyte-like cells from human embryonic stem cells. Cell Transplant 12(1), $1-11$.

Robert Lanza JG, Brigid Hogan, Douglas Melton, Roger Pedersen, E. Donnall Thomas, James Thomson, Ian Wilmut (2009) Essentials of Stem Cell Biology.

Schmidt C, Bladt F, Goedecke S et al. (1995) Scatter factor/hepatocyte growth factor is essential for liver development. Nature 373(6516), 699-702.

Schwartz RE, Linehan JL, Painschab MS, Hu WS, Verfaillie CM, Kaufman DS (2005) Defined conditions for development of functional hepatic cells from human embryonic stem cells. Stem Cells Dev 14(6), 643-655.

Schwartz RE, Reyes M, Koodie L et al. (2002) Multipotent adult progenitor cells from bone marrow differentiate into functional hepatocyte-like cells. J Clin Invest 109(10), 1291-1302.

Seo MJ, Suh SY, Bae YC, Jung JS (2005) Differentiation of human adipose stromal cells into hepatic lineage in vitro and in vivo. Biochem Biophys Res Commun 328(1), 258-264.

Si-Tayeb K, Noto FK, Nagaoka M et al. (2010) Highly efficient generation of human hepatocyte-like cells from induced pluripotent stem cells. Hepatology 51(1), 297-305.

Subramanian K, Owens DJ, Raju R et al. (2014) Spheroid culture for enhanced differentiation of human embryonic stem cells to hepatocyte-like cells. Stem Cells Dev 23(2), 124-131. 
Toivonen S, Lundin K, Balboa D et al. (2013) Activin A and Wnt-dependent specification of human definitive endoderm cells. Exp Cell Res 319(17), 25352544.

Wang F, Zhou L, Ma X et al. (2014) Monitoring of intrasplenic hepatocyte transplantation for acute-on-chronic liver failure: a prospective five-year followup study. Transplant Proc 46(1), 192-198.

Wang P, McKnight KD, Wong DJ et al. (2012) A molecular signature for purified definitive endoderm guides differentiation and isolation of endoderm from mouse and human embryonic stem cells. Stem Cells Dev 21(12), 2273-2287.

Wertheim JA, Petrowsky H, Saab S, Kupiec-Weglinski JW, Busuttil RW (2011) Major challenges limiting liver transplantation in the United States. Am J Transplant 11(9), 1773-1784.

Zorn AM (2008) Liver development. StemBook. Cambridge (MA). 


\section{Figure legends}

Figure 1. Cellular and molecular Evaluation different signaling factors on the generation of DE after 5 days. (A-F) cells treated with differentiation media containing the factors as described. (G and $\mathbf{H})$ undifferentiated DPPSCs, at day 0. Scale bar: $100 \mu \mathrm{m}$. (I-L) quantitative expression of DE- gens under the different treatments after 5 days exposure. GAPDH was used as endogenous control. *p<0.05 vs undifferentiated cells. $¥ \mathrm{p}<0.05$ vs all other conditions. $(n=4)$.

Figure 2. DE induction under different cultural conditions. Cells were cultured on the biomaterials (A) collagen I or (B) fibronectin. Other cultural conditions were (C) FBS free media or (D) co-culture with HepG2 cells. (E-G) the Expression of DE markers are reported in response to the different treatments. GAPDH was used as endogenous control. Scale bars: $100 \mu \mathrm{m} . * \mathrm{p}<0.05$ vs standard protocol. $(\mathrm{n}=4)$.

Figure 3. Molecular and cellular evaluation of DPPSCs mediated hepatocyte-like cells. (A) A time course RT-PCR analysis for the Expression hepatocyte markers, 5 days exposure to Act A. (B) 7 Days exposure to Act A improve DE marker expression. (C) A time course quantitative RT-PCR analysis for the expression of the hepatic markers. GAPDH was used as endogenous control (G-L) cellular morphology at the described differentiation days. (G-I) 100X, (J-I) 400X magnification.

Figure 4. Protein expression of DPPSCs mediated hepatocyte-like cells. (A-C) Immunofluorescence analysis using specific CK18-, ALB-, or AAT antibodies, (A) 100X . (B) and (C) 400X magnification. (D-E) Immunocytochemistry for AFP. (F-G) 
Immunocytochemistry for AAT. (H) Flow cytometry analysis showing the expression of AFP at 11 days of differentiation.

Figure 5. Functional analysis of the generated mature hepatocyte-like cells. Light miscroscope images for undifferentiated DPPSCs or cell with PAS stating for (A) (B) hepatocyte-like cells at day22 of differentiation. (C) CYP3A4 activity measured by luminescence in DPPSCs at days 0 and 22 of differentiation. Time course enzymatic activity of (D) GGT and (E) AST. (F) Time course assay for ALB secretion assay as described in Material and Methods. All measurements are relative to undifferentiated DPPSCs at Day $0, * \mathrm{p}<0.05$.

Figure 6. The experimental protocol for DPPSCs mediated- hepatocyte-like cells generation. Schematic representation of the differentiation protocol including the key manipulated signaling pathways and the different molecular and functional studies performed. 
Figure 1

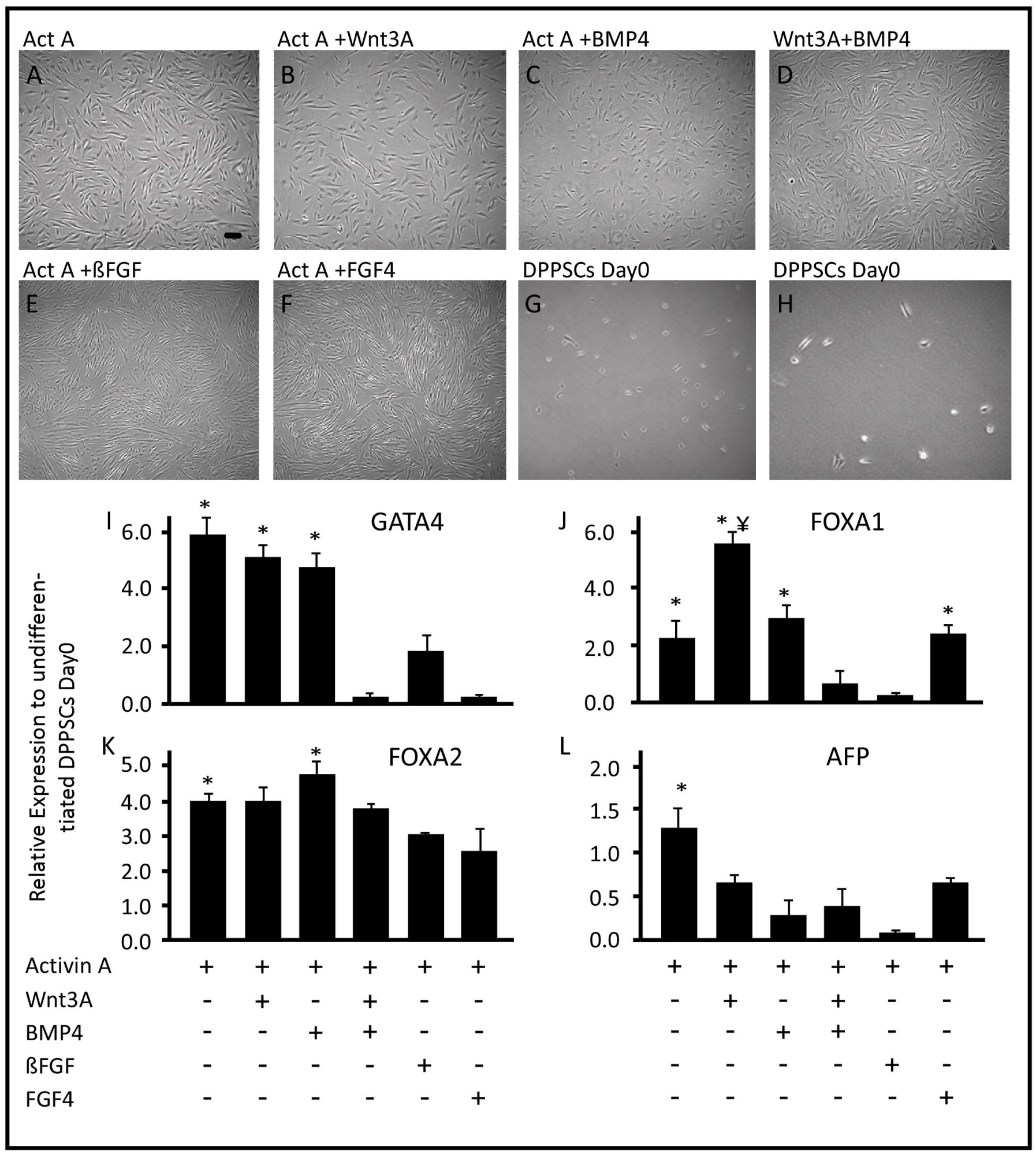


Figure 2

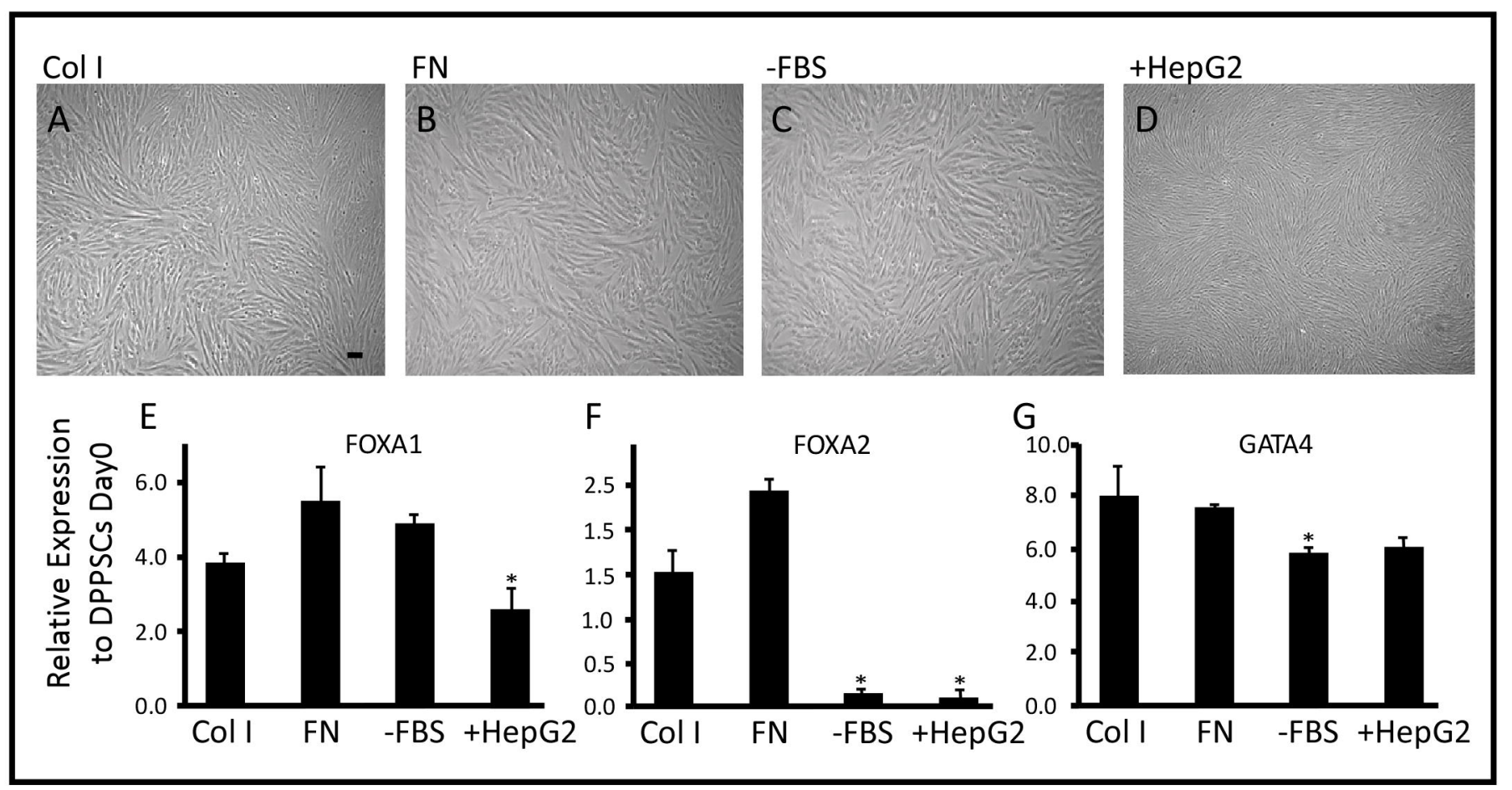


Figure 3

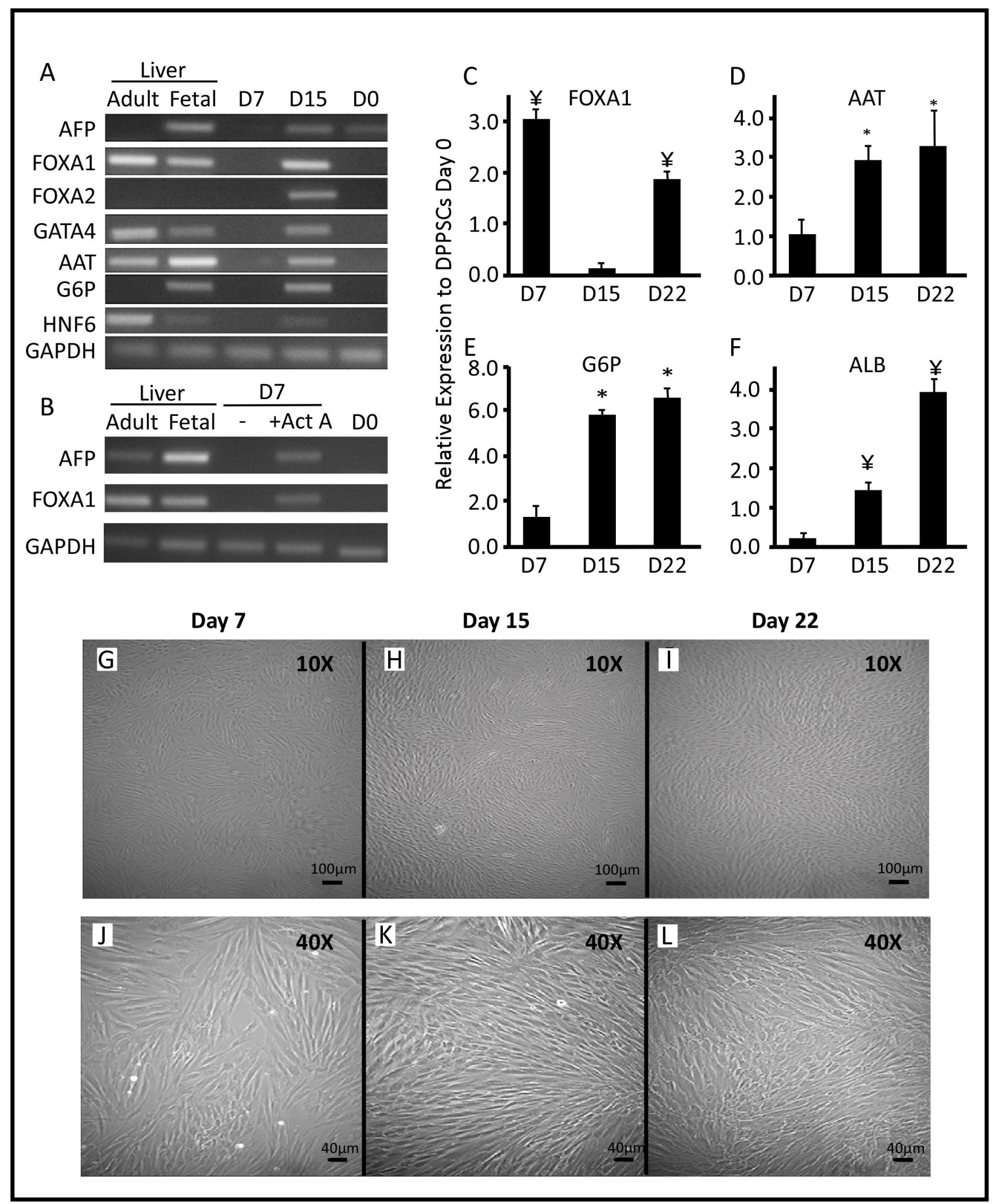


Figure 4

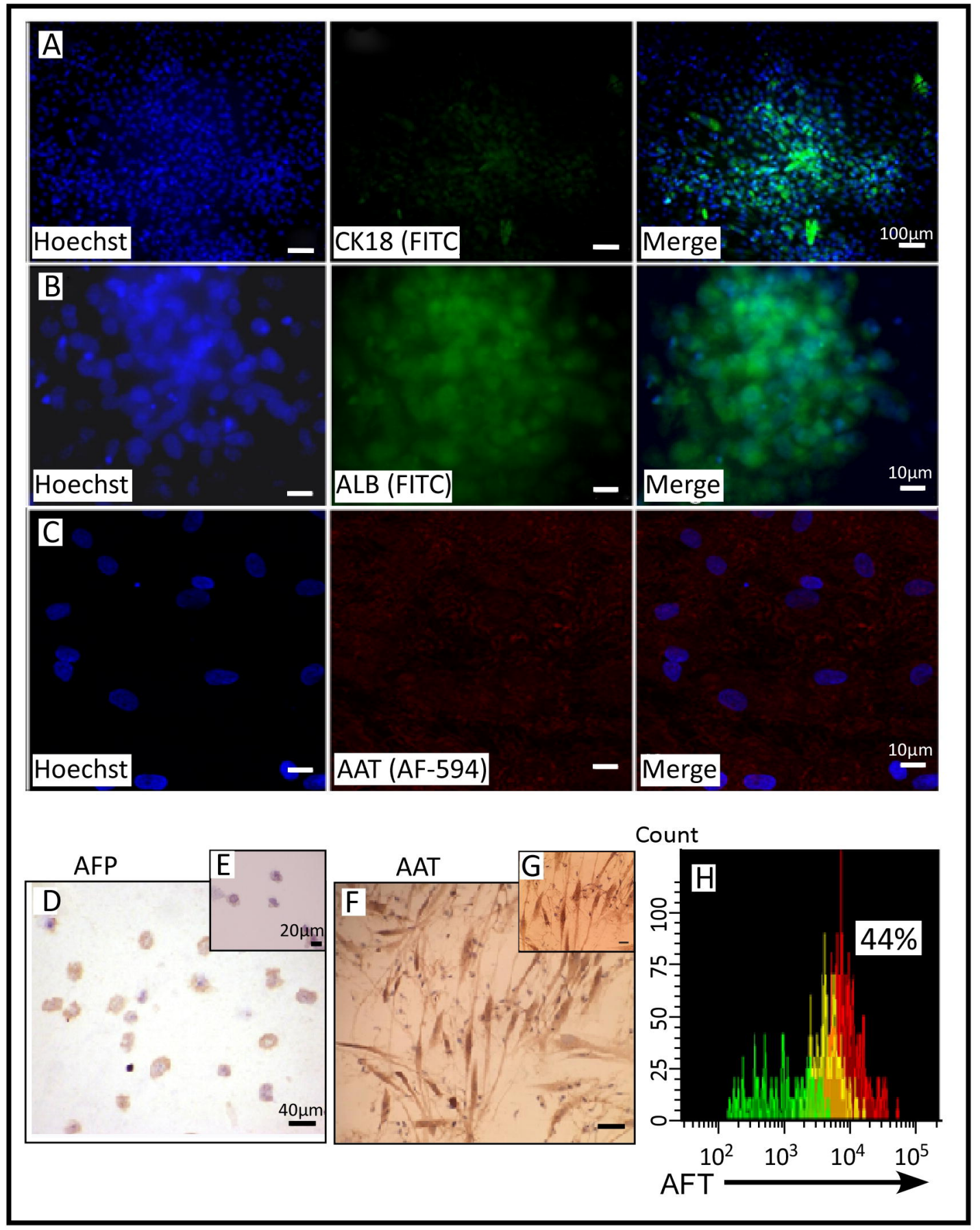


Figure 5

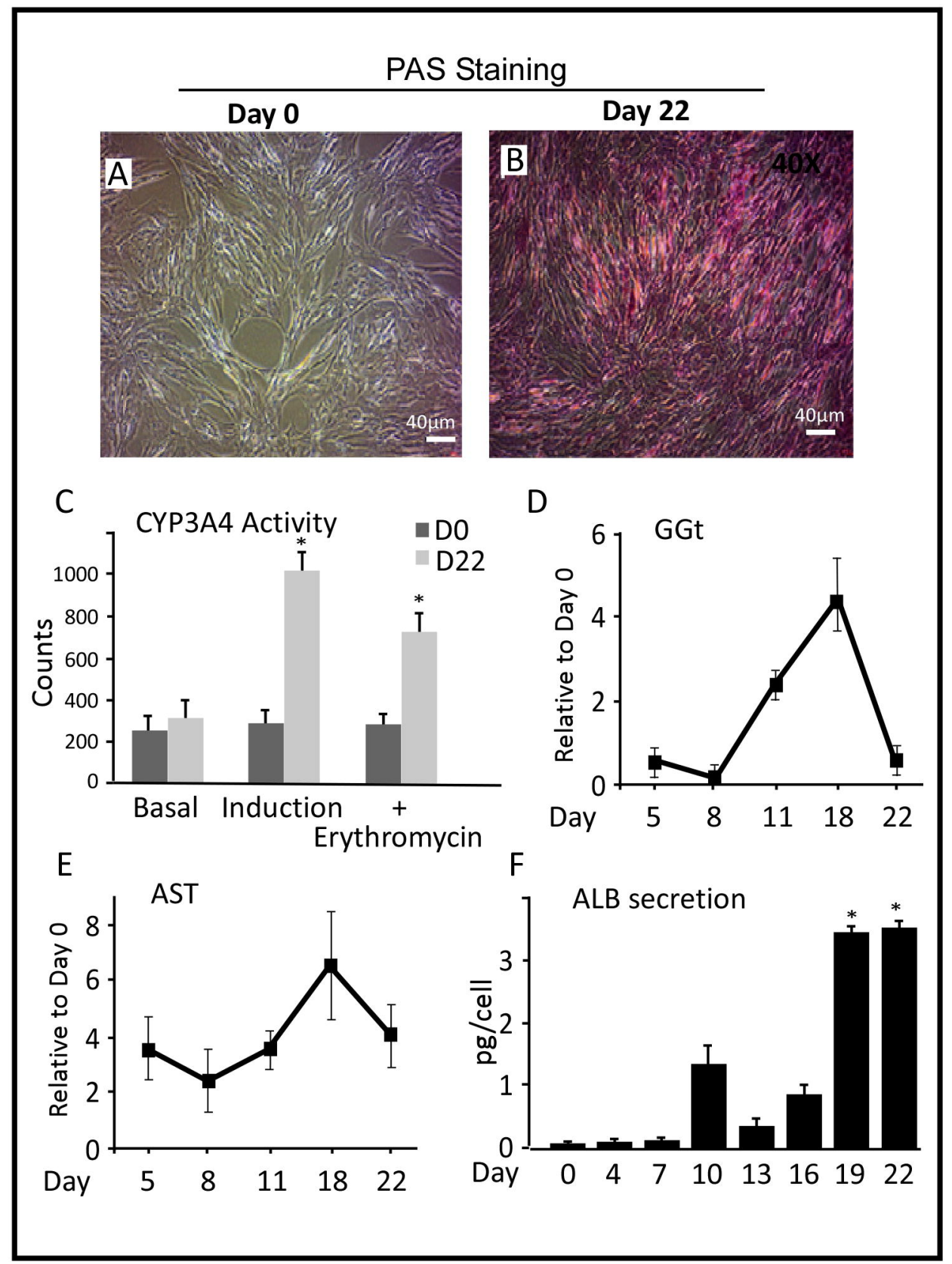


Figure 6

\section{DPPSCs- Mediated Hepatogenesis}

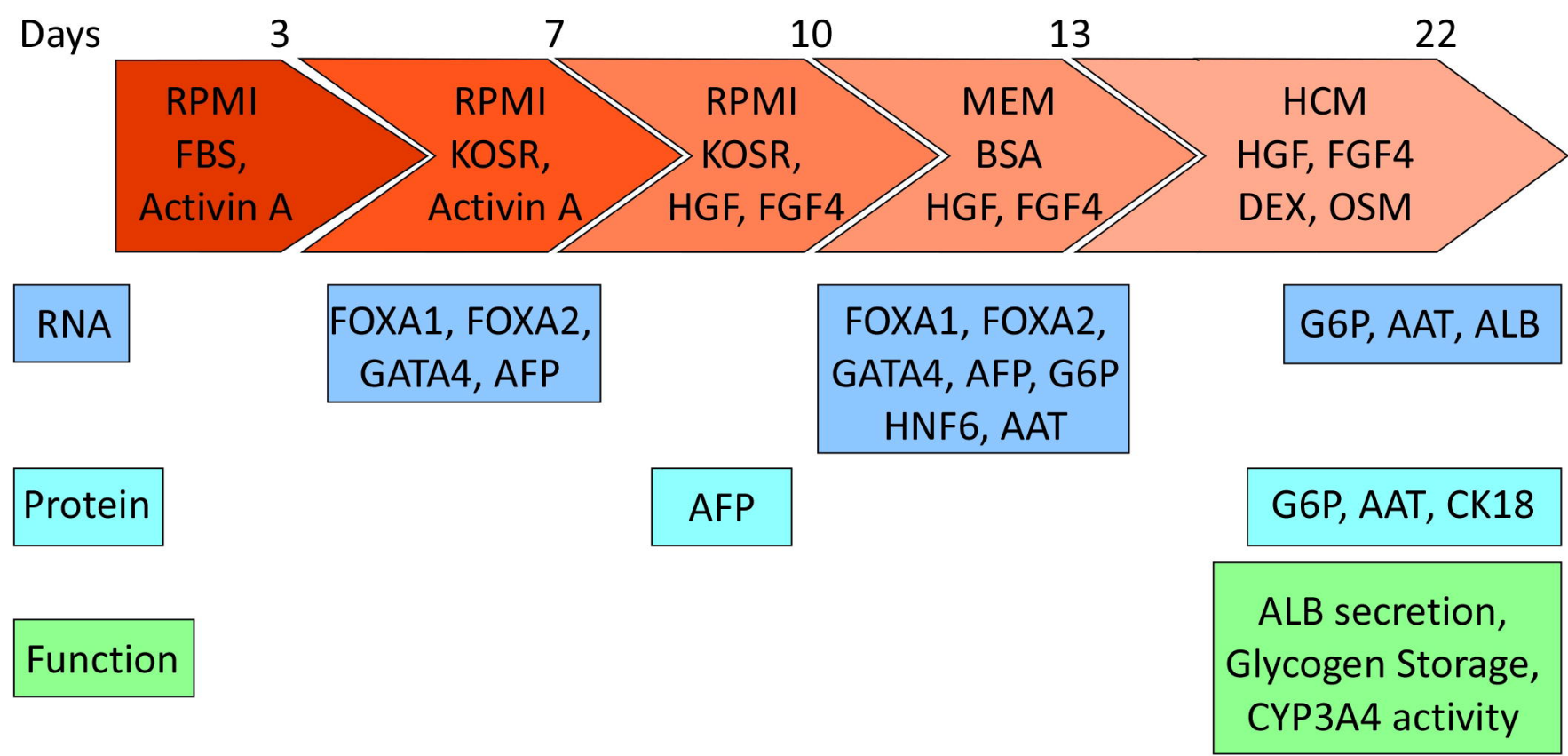

